\section{Two case reports of pneumatosis intestinalis in patients with cancer: is surgical management mandatory?}

\author{
In Hwan Yeo, Yun Jeong Kim
}

Department of Emergency Medicine, Kyungpook National University School of Medicine, Daegu, Korea

Pneumatosis intestinalis (PI) can be classified into two groups. Primary PI is idiopathic, and patients can recover spontaneously. In contrast, secondary PI is considered fatal due to the high mortality rate associated with mesenteric ischemia. Herein, we describe two patients with PI and concurrent pneumoperitoneum. Both patients were receiving targeted anticancer therapy, yet neither developed abdominal pain nor fatal symptoms. One of the patients underwent surgery, while the other was managed conservatively. Even though there were no complications, the patient who underwent surgery was hospitalized for 34 days, whereas the one who was managed conservatively was hospitalized for only five days. Usually, patients with cancer receiving chemotherapy are immunosuppressed and susceptible to infections. Therefore, based on the patients' clinical features, surgical management of patients with cancer who develop PI after receiving anticancer chemotherapy should be done prudently.

Keywords Pneumoperitoneum; Conservative treatment; Neoplasms; Pneumatosis cystoides intestinalis
eISSN: 2383-4625

Received: 17 December 2020 Revised: 17 February 2021

Accepted: 1 March 2021

Correspondence to: Yun Jeong Kim Department of Emergency Medicine, Kyungpook National University School of Medicine, 680 Gukchaebosang-ro, Jung-gu, Daegu 41944, Korea E-mail: kimyjem@knu.ac.kr ORCID

https://orcid.org/0000-0002-7906-9734

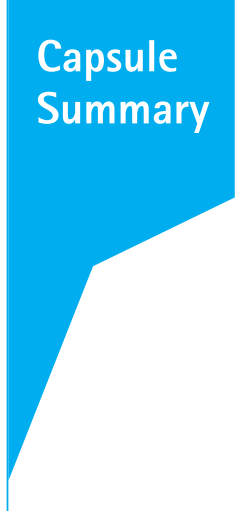

What is already known

Pneumatosis intestinalis can be classified into two groups. Generally, symptomatic patients with mesenteric ischemia can be identified in the emergency department. However, especially in cancer patients, there are several patients with pneumatosis intestinalis who do not have severe symptoms.

\section{What is new in the current study}

We introduce two cancer patients with pneumatosis intestinalis and pneumoperitoneum who had minor symptoms at emergency department presentation. The length of hospitalization was different based on the treatment. Therefore, unnecessary surgical management should be avoided in patients with pneumatosis intestinalis undergoing anticancer chemotherapy.

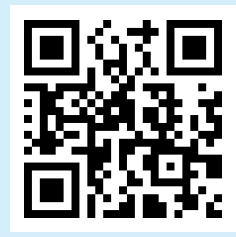

How to cite this article:

Yeo IH, Kim YJ. A case report of pneumatosis intestinalis in patients with cancer: is surgical management mandatory? Clin Exp Emerg Med 2021;8(3):237-241. https://doi. org/10.15441/ceem.20.153

This is an Open Access article distributed under the terms of the Creative Commons Attribution Non-Commercial License (https:// creativecommons.org/licenses/by-nc/4.0/). 


\section{INTRODUCTION}

Pneumatosis intestinalis $(\mathrm{PI})$ is the presence of gas within the wall of the gastrointestinal tract. It is not a clinical diagnosis, but a physical or radiological finding resulting from an underlying pathological process. ${ }^{1} \mathrm{Pl}$ is classified as primary (idiopathic) or secondary. Generally, primary PI is managed conservatively. In contrast, secondary $\mathrm{Pl}$ could be fatal and requires surgery.

Herein, we present two patients diagnosed with $\mathrm{Pl}$ and concurrent renal cell carcinoma (RCC), who presented with abdominal distension after anticancer chemotherapy. In addition, we discussed the treatment of $\mathrm{Pl}$ in patients with cancer.

\section{CASE REPORT}

\section{Case 1}

An 81-year-old male presented to our emergency department (ED) with painless post-prandial epigastric discomfort for five days. He had a 12-year history of RCC, chronic obstructive pulmonary disease, hypertension, and chronic renal failure. The patient was receiving chemotherapy for RCC. A month ago, he has taken $40 \mathrm{mg}$ of methylprednisolone for chronic obstructive pul- monary disease exacerbation for 3 days, and he was on $5 \mathrm{mg}$ of axitinib (Inlyta; Pfizer, New York, NY, USA) daily for the past year. His vital signs were within the normal range. Apart from elevated blood urea nitrogen $(55.5 \mathrm{mg} / \mathrm{dL}, 9.0-23.0 \mathrm{mg} / \mathrm{L})$ and creatinine $(1.7 \mathrm{mg} / \mathrm{dL}, 0.7-1.3 \mathrm{mg} / \mathrm{dL}$ ) levels, his blood tests (complete blood count with differential count, liver function tests, coagulation tests, electrolytes, and lactate level, etc.) were within normal ranges. Plain abdominal radiography and abdominal computed tomography (CT) without contrast revealed diffuse $\mathrm{Pl}$, predominantly in the jejunum, with a large amount of intra-abdominal free air (Fig. 1). Following a suspicion of bowel perforation, an exploratory laparotomy was performed. However, during the operation, we only found $\mathrm{Pl}$, which occupied $20 \mathrm{~cm}$ of the bowel wall 1 $\mathrm{m}$ away from the ileocecal valve without ischemic changes or bowel perforation (Fig. 2). On postoperative day (POD) 4, the patient started eating soft foods and did not develop any complications. On POD 8, plain abdominal radiography was performed, which showed the absence of any free air. However, the patient developed diarrhea after resuming foods. On POD 17, abdominal CT was repeated, which showed the absence of PI or pneumoperitoneum. The patient was discharged on POD 34 without any complications.
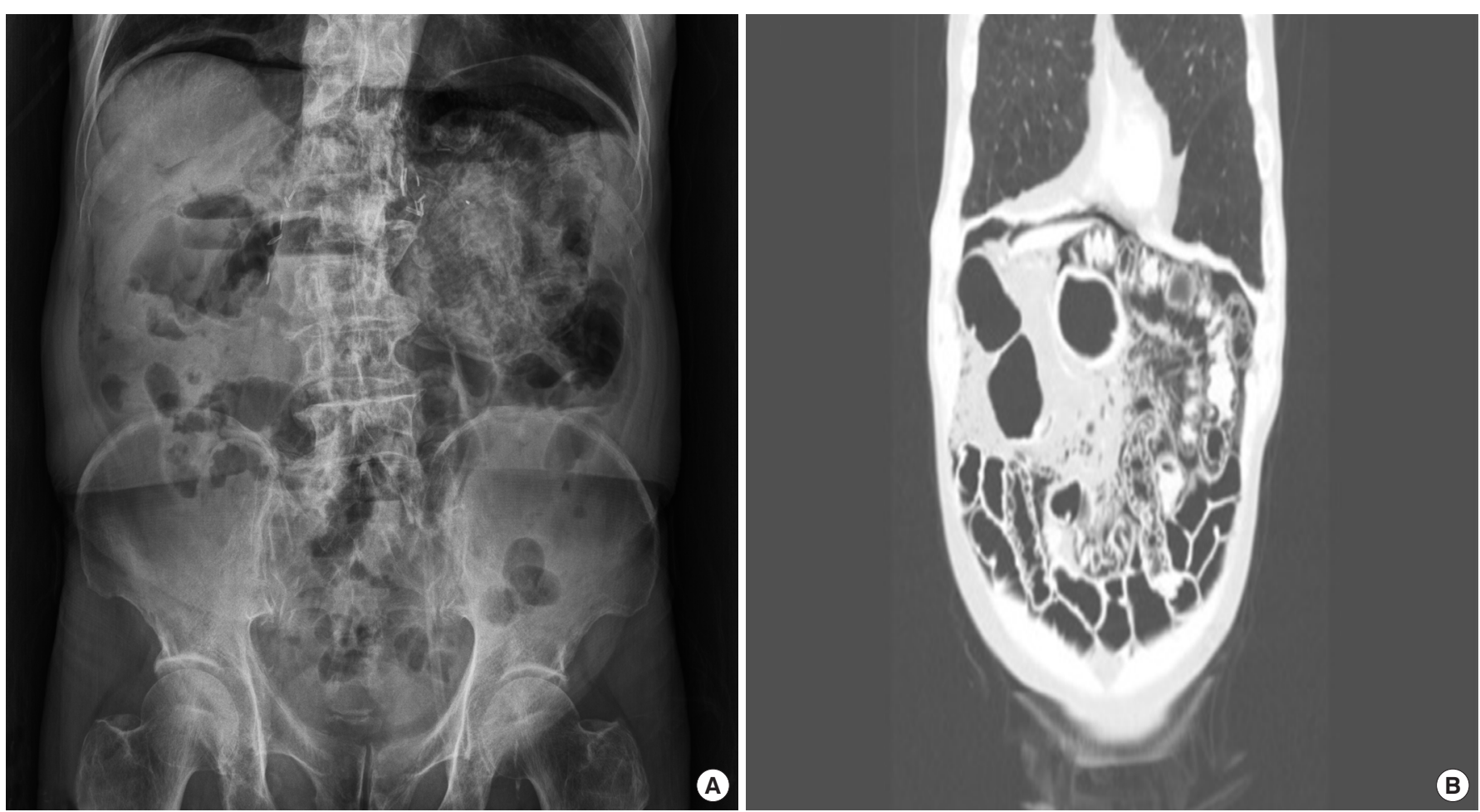

Fig. 1. Initial plain abdominal X-ray and abdominal computed tomography images. (A) The simple abdominal erect image shows subphrenic free air and pneumatosis intestinalis in the left upper quadrant. (B) The coronal abdominal computed tomography image shows pneumoperitoneum and pneumatosis intestinalis in the left upper quadrant. Informed consent for publication of the clinical images was obtained from the patient. 

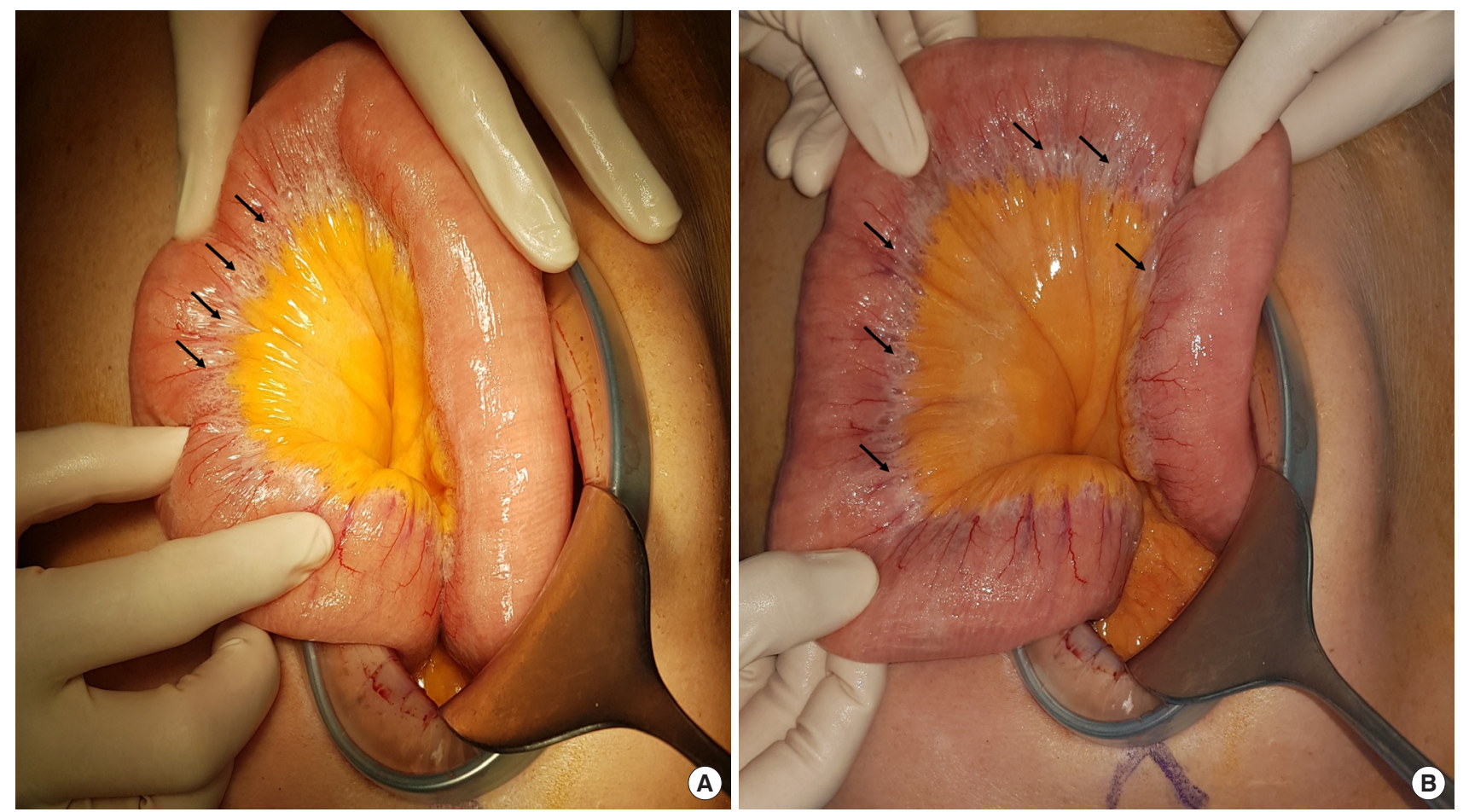

Fig. 2. Gross photographs of pneumatosis intestinalis in the operation field. The arrows in the figure indicate pneumatosis intestinalis. (A) and (B) lleum. Informed consent for publication of the clinical images was obtained from the patient.
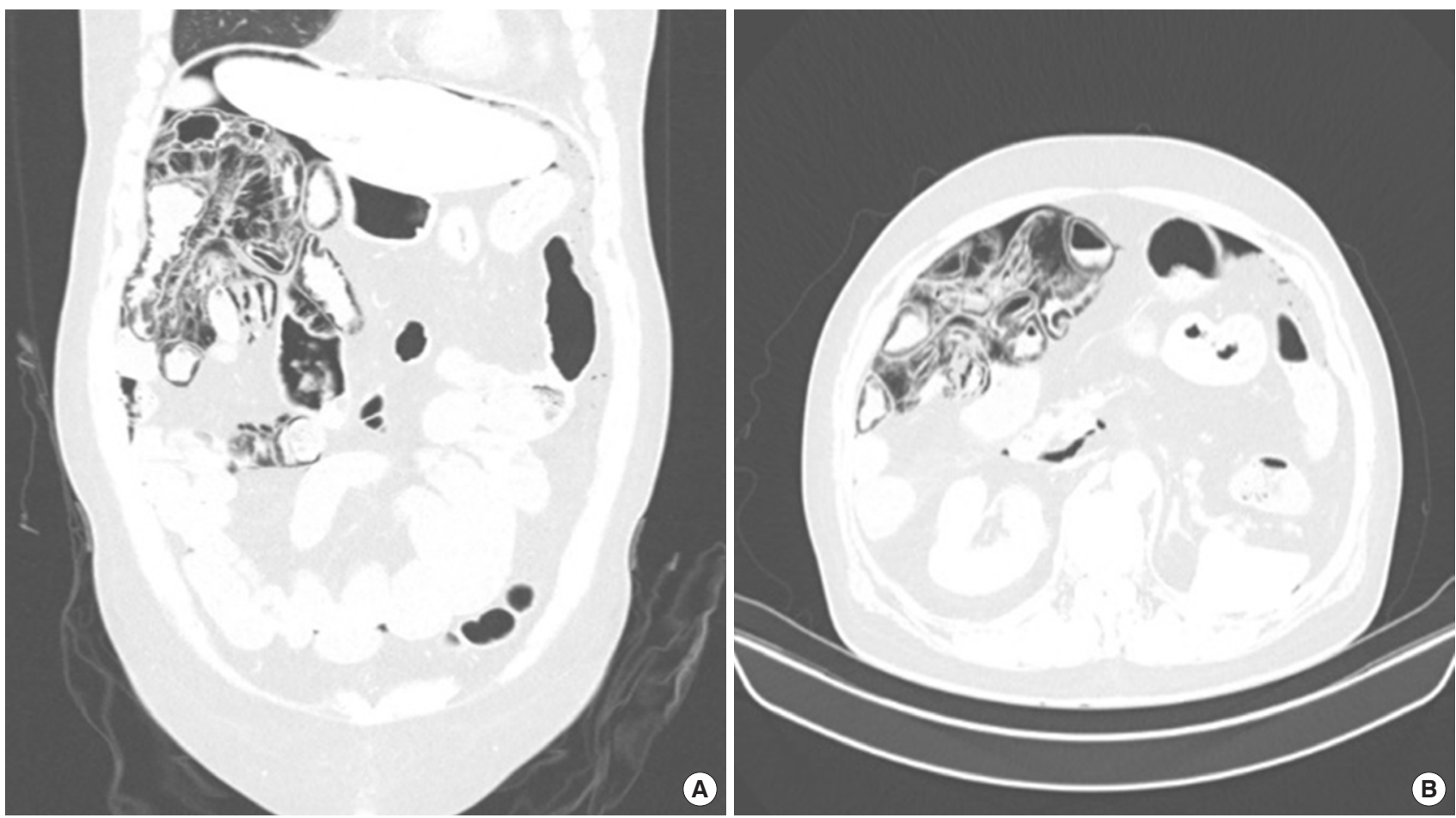

Fig. 3. Initial abdominal computed tomography (CT) images of a 63-year-old male incidentally diagnosed with pneumatosis intestinalis (PI). (A) The coronal $\mathrm{CT}$ image shows pneumoperitoneum and PI in the small intestines. (B) The axial CT image shows pneumoperitoneum and PI in the small intestines. Informed consent for publication of the clinical images was obtained from the patient. 


\section{Case 2}

A 63-year-old male was referred to the ED by a radiologist who found $\mathrm{PI}$ and pneumoperitoneum incidentally on CT during his regular check-up. He complained of a migrating discomfort in both flanks and abdominal distension, which started a month prior to this consultation. He had a medical history of RCC and hypothyroidism. The patient was receiving chemotherapy for RCC. He had been taking $40 \mathrm{mg}$ of cabozantinib (Cabometyx; Exelixis, Alameda, CA, USA) daily for the past year. His initial vital signs were within the normal range. He had elevated blood urea nitrogen $(27.3 \mathrm{mg} / \mathrm{dL})$ and creatinine $(1.81 \mathrm{mg} / \mathrm{dL})$ levels. However, other blood tests including lactate level were within normal ranges. Plain abdominal radiography and abdominal CT without contrast revealed diffuse $\mathrm{Pl}$, predominantly in the jejunum, with free air in the perihepatic space (Fig. 3). He was hospitalized and managed conservatively. He started eating soft foods on postadmission day 3. He was discharged on postadmission day 5 without complications.

\section{DISCUSSION}

$\mathrm{Pl}$ is caused by several underlying gastrointestinal or extra-gastrointestinal diseases, such as autoimmune diseases (e.g., scleroderma), inflammatory diseases (e.g., inflammatory bowel disease), infectious diseases (e.g., Clostridium difficile infection or human immunodeficiency virus), pulmonary diseases (e.g., chronic obstructive pulmonary disease), drugs (e.g., corticosteroids and immunosuppressive agents), and trauma (e.g., blunt abdominal trauma). ${ }^{1,2}$ Studies suggest that PI occurs due to disruption of the mucosal integrity. There are two major theories to explain the cause of the intramural gas ${ }^{3}$ : (1) the bacterial theory: gas-producing bacteria translocate from the gastrointestinal lumen to the submucosal space through mucosal gaps or areas of the enhanced permeability and (2) the mechanical theory: normal gas moves from the lumen into the non-inflamed bowel wall due to disruption of the mucosal integrity.

Primary PI (15\%) is asymptomatic, rare, and usually located in the colon. Individuals who are at a high risk of primary $\mathrm{Pl}$ are males aged from 40 to 60 years. ${ }^{4}$ Secondary $\mathrm{Pl}$ is more common and can be fatal; it is associated with several underlying conditions, such as coronary artery disease, peripheral vascular disease, and smoking, which could lead to acute mesenteric ischemia. ${ }^{5}$ A case series of 919 patients with PI showed that PI had a peak incidence among people in their 40s, with a male predominance (male-tofemale ratio 3:1). PI affected all parts of the gastrointestinal tract: small bowel (42\%), large bowel (36\%), and both (22\%). ${ }^{6}$ Patients with cancer usually receive immunosuppressive therapies, which can reduce the number of lymphocytes in the gastrointestinal wall, particularly in Peyer's patches, and thereby impairing the bowel defense barrier system. ${ }^{7}$ In a case series of 84 cancer-related PI cases, the CT findings associated with clinically worrisome PI were portomesenteric venous gas (PVG), bowel dilatation, bowel wall thickening, ascites, and peri-intestinal mesenteric stranding, whereas PI confined in the colon was benign. ${ }^{3}$

In a previous report of three $\mathrm{Pl}$ cases, an association between $\mathrm{PI}$ and steroids or chemotherapeutic agents (e.g., lapatinib, capecitabine, zoledronic acid, docetaxel, etc.) was found, and all the cases were benign. Such cases can be resolved with supportive care alone and close observation. ${ }^{8}$ The most common cause of nonsurgical pneumoperitoneum is $\mathrm{PI}^{9,10}$ When a cyst ruptures, it may result in a pneumoperitoneum with a spectrum of symptoms ranging from asymptomatic to acute abdominal pain. Even when it mimics secondary $\mathrm{Pl}$, patients with gross radiological evidence could follow a more benign clinical course. ${ }^{10,11}$ Thus, when there are no abdominal signs of peritonitis and the patient is afebrile and has normal white blood cell counts, conservative management should be considered. ${ }^{11}$

In case 1, the patient had received targeted anticancer therapy using axitinib. In this patient, PI was confined to the small intestines with pneumoperitoneum; he did not have abdominal pain or any evidence of bowel ischemia. Although he did not have potentially fatal symptoms, such as severe abdominal pain, acidosis, and fever, which are frequently seen in secondary $\mathrm{PI}$, the general surgeon performed an exploratory laparotomy to rule out bowel perforation. After confirming the absence of bowel perforation, the surgeon concluded that the patient should be managed conservatively. Due to his immunosuppressive status, old age, and imaging results, the surgeon was skeptical of the patient's condition; however, conservative management and follow-up observation were successful.

Case 2 was similar to case 1 . The patient was receiving targeted anticancer therapy. In case 2, the PI was confined to the small bowel with pneumoperitoneum, and the patient did not have pain. His symptoms were not worrisome. He was managed conservatively during his 5-day hospitalization.

Clinicians must carefully decide how to treat a patient with $\mathrm{PI}$, especially those with cancer. In a case series of 88 patients with $\mathrm{Pl}$, the patients' characteristics and treatments were reviewed, and a management algorithm was constructed..$^{5}$ Among the 84 patients, 70 patients were included in an exploratory series, while 14 were included in a confirmatory series. Among the 70 patients, 19 had benign disease, and six of them with PI received chemotherapy. Among them, ten patients were managed conservatively, and nine patients underwent exploratory laparoscopy. There were 
no signs of bowel injury, ischemia, or necrosis. A management algorithm was constructed using the results of the exploratory series. A 3-step algorithm consists of the patients' stability (critically ill and unstable), radiologic findings (PI/PVG with mechanical disease or iatrogenic gastrointestinal trauma), and vascular disease score (determined by patients' medical history, physical examination, lactate level, and radiologic findings; a score below 4 suggests benign PI). Seven of 14 patients in the confirmatory series had benign $\mathrm{Pl}$; all their vascular disease scores were below 4 . One patient underwent non-therapeutic laparoscopy, and the postoperative period for all the patients was uneventful.

Furthermore, based on the management algorithm for PI and/ or PVG, we can classify the PI in our patients as benign. They were not critically ill or unstable, and they had no vascular obstructions or iatrogenic GI trauma. In addition, the vascular disease score was 2.5 and 1.5 , respectively.

The management of patients with PI remains controversial. In patients with $\mathrm{Pl}$ and concurrent pneumoperitoneum, clinicians cannot be certain of the presence of bowel perforation. In addition, surgical exploration is not a simple procedure wherein a patient can recover completely several hours after the operation. For this reason, clinicians should thoroughly review PI cases, apply a management algorithm to patients with $\mathrm{Pl}$, and then carefully select a treatment option. If the patient has benign PI, clinicians should observe their patients closely. If the patient has secondary $\mathrm{PI}$, surgical management should be provided immediately. Non-surgical treatment of benign PI can improve patients' health and shorten their hospitalization time. Physicians should consider primary PI associated with anticancer chemotherapy when a patient presents with vague symptoms at the emergency department. Careful consideration of the patient's history, thorough examination, and assessment of diagnostic clues is mandatory.

\section{CONFLICT OF INTEREST}

No potential conflict of interest relevant to this article was reported.

\section{REFERENCES}

1. St Peter SD, Abbas MA, Kelly KA. The spectrum of pneumatosis intestinalis. Arch Surg 2003;138:68-75.

2. Vogel Y, Buchner NJ, Szpakowski M, Tannapfel A, Henning BF. Pneumatosis cystoides intestinalis of the ascending colon related to acarbose treatment: a case report. J Med Case Rep 2009;3:9216.

3. Lee KS, Hwang S, Hurtado Rua SM, Janjigian YY, Gollub MJ. Distinguishing benign and life-threatening pneumatosis intestinalis in patients with cancer by CT imaging features. AJR Am J Roentgenol 2013;200:1042-7.

4. Koss LG. Abdominal gas cysts (pneumatosis cystoides intestinorum hominis); an analysis with a report of a case and a critical review of the literature. AMA Arch Pathol 1952;53:52349.

5. Wayne $E_{\text {, Ough }} M$, Wu $A$, et al. Management algorithm for pneumatosis intestinalis and portal venous gas: treatment and outcome of 88 consecutive cases. J Gastrointest Surg 2010;14:437-48.

6. Jamart J. Pneumatosis cystoides intestinalis: a statistical study of 919 cases. Acta Hepatogastroenterol (Stuttg) 1979;26:41922.

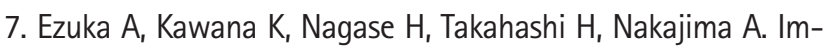
provement of pneumatosis cystoides intestinalis after steroid tapering in a patient with bronchial asthma: a case report. J Med Case Rep 2013;7:163.

8. Sassi C, Pasquali M, Facchini G, Bazzocchi A, Battista G. Pneumatosis intestinalis in oncologic patients: when should the radiologist not be afraid? BJR Case Rep 2016;3:20160017.

9. Mularski RA, Ciccolo ML, Rappaport WD. Nonsurgical causes of pneumoperitoneum. West J Med 1999;170:41-6.

10. Iflazoglu N, Gokce ON, KIvrak MM, Kocamer B. Spontaneous idiopathic pneumoperitoneum with acute abdomen. Ulus Cerrahi Derg 2013;31:110-2.

11. Williams NM, Watkin DF. Spontaneous pneumoperitoneum and other nonsurgical causes of intraperitoneal free gas. Postgrad Med J 1997;73:531-7. 